\title{
Spatiomotor cueing in unilateral left neglect: three case studies of its therapeutic effects
}

\author{
Ian H Robertson, Nigel T North, Colin Geggie
}

\begin{abstract}
Limb activation contralateral to a cerebral lesion seems to reduce visual neglect, though whether this is due to perceptual cueing or hemispheric activation is controversial. Three case studies are presented which attempt to use this experimental finding therapeutically in the rehabilitation of unilateral left neglect. The first study used a combination of perceptual anchoring training with left arm activation procedures and produced improvements. The second used the same method, but stimulated left arm activation using an avoidance conditioning procedure, again with positive results. The third case treatment focused on cueing for left arm activation without explicit instructions for perceptual anchoring, with positive results.
\end{abstract}

\section{$(\Im$ Neurol Neurosurg Psychiatry 1992;55:799-805)}

Joanette and Brouchon ${ }^{1}$ described a 64 year old woman who suffered a right brain stroke and who pointed to stimuli on her left as if she had seen them on her right. ${ }^{12}$ Interestingly, there was an interaction between the side of space in which the stimulus appeared and the arm which was used. Only when the right arm was used in response to a left sided stimulus did the allaesthesia appear. When the other arm was used in response to the same stimulus on the same side, there was no allaesthetic response. A subsequent series of cases found that not only allaesthetic problems revealed such an interaction. ${ }^{3}$ In a standard stimulus identification procedure, neglect was less severe when the limb contralateral to the lesion was used to point to the target stimuli than when the limb ipsilateral to the lesion was used. In a single case study, Halligan and Marshall also found that using the left arm for cancellation and line bisection tasks resulted in less neglect. ${ }^{4}$

These findings are in line with the theoretical propositions of Rizzolatti and Camarda, who proposed that spatial attention is based on a series of circuits largely independent from one another which programme motor plans in a spatial framework. Spatial attention is not seen as a supraordinate function controlling whole brain activity, but as a property intrinsically linked to the premotor activity and distributed among a range of centres.

Subsequently, however, Halligan et al showed in a series of experiments that the advantage of arm use in reducing neglect was better explained by a spatiomotor cueing process than by an hemispheric activation hypothesis. ${ }^{6}$ More specifically, they found that the advantage of left arm use in line bisection was eliminated by having the subjects begin the task on the right side of the line - that is, with the arm crossed beyond the body midline. This, however, does not exclude the possibility that any hypothesised activation effect may depend on limb activation within left hemispace, as opposed to limb activation per se.

There is a further reason for suggesting that recruitment of the limb contralateral to the lesion might be effective in treatment. This rests on the assumption that previous treatments of unilateral neglect have produced effects that tend to generalise only to situations similar to the training procedures. ${ }^{7}$ This in turn has been attributed to compromised nonlateralised attentional deficits among patients with neglect. ${ }^{8}$ In short, chronic neglect patients are capable of learning specific compensatory scanning responses to specific stimuli, but much less capable of learning general scanning strategies.

This leads to a clear clinical problem in the rehabilitation of unilateral left neglect: is there a stimulus that is reliably present in all the different situations in which the sufferer must operate? One answer to this is the person's left arm. In short, the question posed in these studies is whether left arm activation and perceptual anchoring can produce enduring and therapeutic improvements in neglect.

A caveat to this approach is the high incidence of hemiplegia associated with unilateral neglect. Robertson, however, has shown one case of severe left hemiplegia where using the hemiplegic left arm aided by the right arm and including some minimal left shoulder movement resulted in a significant reduction in inattention to left arm compared with standard right arm performance. ${ }^{9}$ Furthermore, in two of the three cases reported below, patients did have severe hemiplegias: the movements required were the kind of residual minimal responses that are not uncommon in hemiplegic patients. Finally, even where there is no movement whatsoever in the left arm, the possibility of using the left arm as a passive perceptual anchor remains.

\section{Case reports}

CASE 1

Subject

The subject was a 30 year old, right handed man who was found unconscious at the bottom 
of a flight of stairs, having fallen down them when intoxicated. He suffered a right frontoparietal haematoma and had a Glasgow coma score of 5 on admission. His pupils were equal and reacting, but he had a dysconjugate gaze. A right frontotemporoparietal craniotomy was performed after a CT scan revealed a moderately sized acute subdural haematoma on the right side with midline shift. He had been employed as a labourer in a brewery but had been made redundant several weeks before the injury occurred. His estimated premorbid IQ (national adult reading test) was 107 .

Nine weeks later he was admitted to the Astley Ainslie Rehabilitation Hospital, still confused, with a mini-mental state test score of 13 out of 30 , indicating severe cognitive deficits. He was confused and disoriented. A CT scan performed 22 weeks after injury revealed atrophy of the entire right cerebral hemisphere, in the presence of a normal left cerebral hemisphere. (The subject had no history of treatment for alcohol problems and had held down a regular labouring job for several years; hence it is unlikely that alcohol consumption had a significant effect on this degree of cerebral atrophy, although it cannot be ruled out.)

Neuropsychological testing at this time found the patient showing marked unilateral visual neglect: scoring only 5 out of 47 on copy of Rey figure; scoring 15 out of 104 on a letter cancellation test ${ }^{10}$; reading only the rightmost $20 \%$ of a 26 item prose reading test; missing all details on the left side when drawing a flower from memory.

On the Wechsler memory scale logical memory subtest he remembered only six out of $\mathbf{4 5}$ items on immediate recall, he remembered none on delayed recall, where he showed marked confabulation. He could remember a maximum of five digits forward and three backward. On the revised Wisconsin card sorting test (Nelson version) he made 16 errors, 12 of which were perseverative, suggesting the presence of frontal lobe-type deficits. On Benton's word fluency test (giving words with letters beginning $F, A$, and $S$ ) he managed only 15 in three minutes, definitely an impaired performance.

The patient suffered a left hemiparesis with increased tone and reduced sensation in both the left arm and leg, as well as decreased proprioception in his left foot. The power in his left arm was assessed as $1 / 5$, that in his left leg as $2 / 5$. He also showed telegraphic speech and had a left homonymous hemianopia.

\section{Baseline assessment}

To obtain a baseline of his visual neglect, the following measures of neglect were taken several times, the number varying slightly across measures because of administrative difficulties: letter cancellation (10 measures) (cancel $c$ and e); telephone dialling (9 measures) (subtest of behavioural inattention test $^{11}{ }^{12}$-requiring the subject to dial three numbers $(5,6$, and 7 digits) printed on cards in front of him or her), and reading from a passage of around 70 words (11 measures).
Parallel versions of these tests were given over a period of six weeks before the start of training. In addition, a control measure-backward digit span-was given on 11 occasions in the baseline phase. The purpose of this measure was to control for spontaneous remission as a cause of any observed changes in behaviour.

There followed an eight week post-baseline phase in which six sets at a time of the above measures were taken independent of the training sessions, usually in the morning before an afternoon training session.

\section{Training procedure}

The aim of training was to teach the subject always to place and hold his left arm at the left margin of any activity he was involved in. $\mathrm{He}$ then had to always "find" his left arm-that is, locate it visually - before beginning what he had to do.

At first he had great difficulty in keeping his arm in position once his attention was occupied by the task in hand (washing, eating, reading, for example). With feedback, however, he began to learn to do this while also engaging in these other tasks. This training was given by a psychologist for one hour each day for the 11 working days of the training period.

Nursing staff also participated in the training by cueing the patient to "anchor" with his left arm when eating, washing, and shaving, a total input of approximately two hours a day. Occupational therapists used the same strategy during the hour long daily therapy sessions during the training period. Therapy therefore took place as part of a wide range of rehabilitation activities and was very intensive (a total of cued practice of four hours a day over 11 days, giving a total of 44 hours of training).

A non-parametric statistical test (MannWhitney U test) was used to compare baseline and post-baseline performance for each measure in each of the three cases reported.

\section{Results}

Letter cancellation performance improved from a mean (SD) of $16.3(2.4)$ correctly cancelled letters before treatment to a mean of $47.5(8 \cdot 1)$ during training (fig $1, p<0.001$ ). The prose reading performance also improved from $52 \%(18 \%)$ accuracy during baseline to a mean of $93 \%(7 \%)$ accuracy during training (fig $1, \mathrm{p}<0.001$ ).

Backward digit recall did not improve in the training period $(3.2(0.6) v 2 \cdot 7(0.52))$, suggesting that, at least as far as this measure is concerned, there was no spontaneous remission (fig 2, $\mathrm{p}>0 \cdot 1$ ). Telephone dialling did improve from a mean of $9 \cdot 2(2 \cdot 0)$ correctly dialled numbers at baseline to a mean of 16.0 $(1.4)$ in the training phase (fig $2, \mathrm{p}<0.001$ ).

\section{CASE 2}

The previous case suggested that, where there is some residual control over a hemiparetic arm, it may be possible to train a patient with visual neglect to use the arm as a perceptual anchor to aid scanning over the full visual field. 


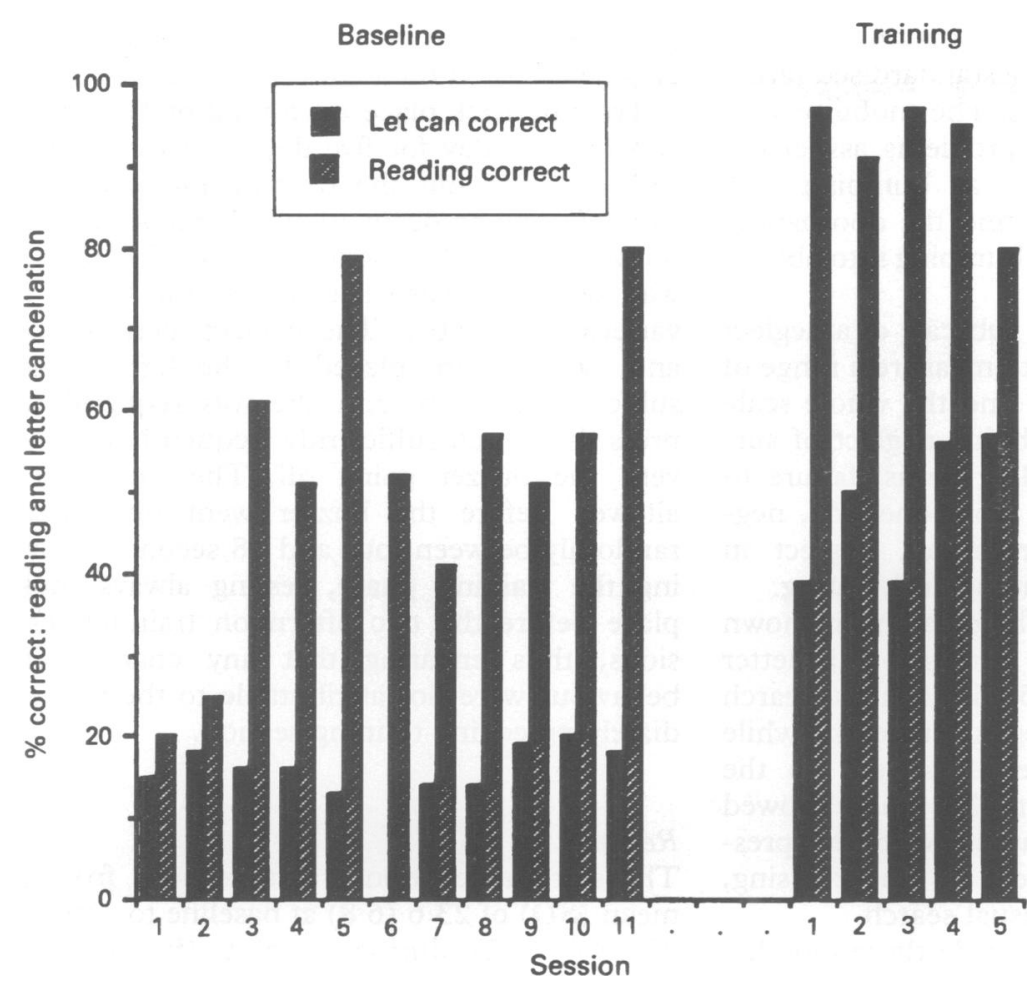

Figure 1 Case 1: letter cancellation and reading, at baseline and after training.

The advantage of this procedure is that the perceptual anchor is, by definition, permanently available to the patient and does not have to be internalised or mentally imposed on the task in hand. The activation required of the arm may result in increased activation of certain perceptuomotor circuits whose malfunctioning was responsible for the left visual neglect. It is impossible, however, to disentangle the effects of the activation from those of perceptual anchoring in case 1.

We attempted to overcome a number of problems with the first cast study in case 2 .

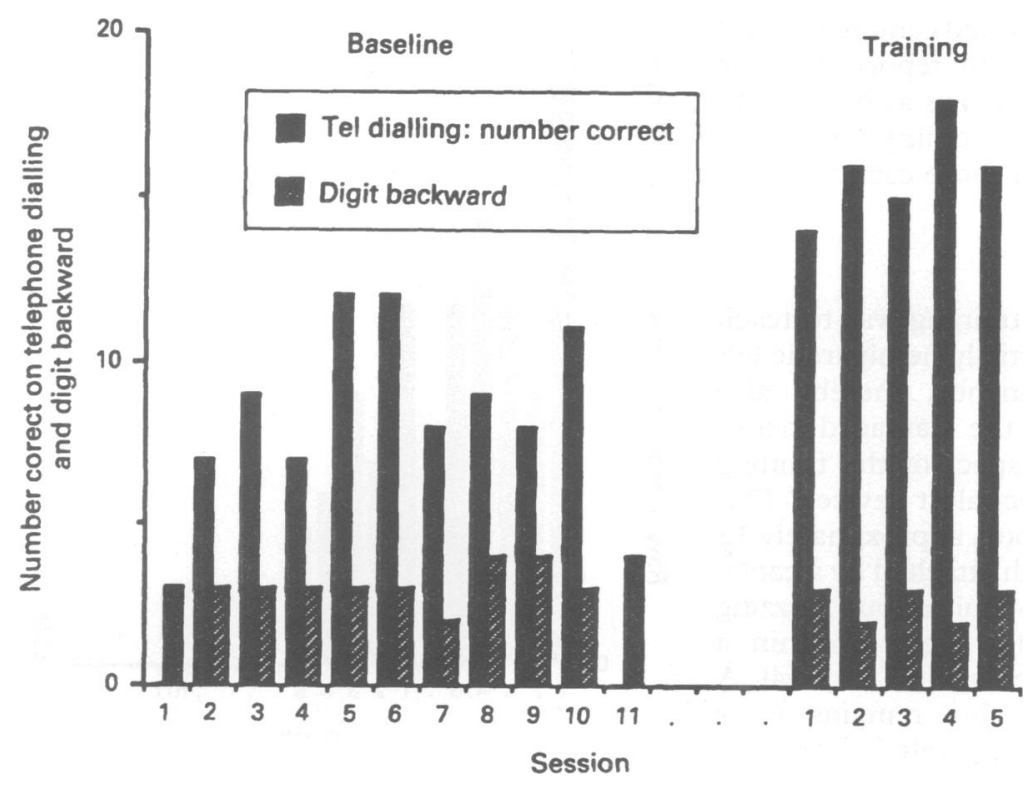

Figure 2 Case 1: telephone dialling and digit backward, at baseline and after training.
Firstly, the amount of therapy was considerable in the first study. Could the same results be obtained with less therapeutic intervention? Therapy was reduced to two hours a day for five days, giving a total of 10 hours. Secondly, assessment of neglect immediately after the intervention phase was carried out blind by a psychologist unaware of the nature of the study. Thirdly, the patient's husband carried out daily ratings of the patient's everyday problems; he was blind as to the nature of the treatment or of the nature of the study. Fourthly, six follow up sessions over a period of four weeks after training were implemented, during which time the patient's husband continued to keep daily recordings of everyday problems with neglect.

Perhaps the most important change, however, was the instigation of an avoidance conditioning device ("neglect alert device"), developed by IR and CG specifically to improve learning in the training regimen used in Case 1. This and the other changes are described in greater detail below.

\section{Subject}

Case 2 was a 61 year old housewife who had had hypertension for 20 years. Thirty six hours after mastectomy for a carcinoma in situ without node involvement, she had suffered a right sided stroke. A CT scan was not available. Neurological examination carried out on admission to the Astley Ainslie Rehabilitation Hospital in November 1990 showed a left sided hemiparesis with associated mild spasticity. Neurological assessment found that power was $0 / 5$ in her left hand, $3 / 5$ in her left shoulder and elbow, and 5/5 in her left leg. She was reported to have a loss of proprioception in her left arm and leg. She also had a left homonymous hemianopia, with her cranial nerves being otherwise intact. She showed no dyspraxia, dysphasia, or tremor nor any cerebellar signs. She was capable of standing with the help of one person and was independent in dressing. She had very severe left sided visual and sensory inattention on neurological assessment. At 11 weeks after stroke her neglect was severe and handicapping, and therapists reported that she was making no progress in therapy because of her neglect.

On formal neuropsychological assessment, the patient showed severe left visual neglect, as measured by the behavioural inattention test (BIT). She showed severe neglect on 14 of 15 subtests of this scale and was in the severe neglect category on both "behavioural" (score $33 / 81$ ) and "conventional" (56/146) tests. The patient was fully oriented in time, place, and person and scored 23/30 on the mini-mental state examination. Her forward digit span was five and her backward span three.

\section{Baseline assessment}

Baseline measures were collected for three, four, or five sessions over a period of five days, depending on the measure. The star cancellation subtest of the BIT ${ }^{112}$ was given on five consecutive days, Monday to Friday. In addition, the patient's husband carried out daily 
ratings ("frequently", "occasionally," or "never") on a subscale of a standardised rating scale for neglect problems. The mobility subscale related to mobility problems associated with visual neglect, such as bumping into doorways, an inability to find the doorhandle when opening doors, and bumping into objects or people on one side.

This rating scale was a subscale of a neglect questionnaire developed to measure a range of everyday life functions, ${ }^{13}$ and the whole scale had seven subscales; mobility, neglect of surroundings, difficulty finding items, failure to respond when addressed from one side, neglect in dressing and grooming, neglect in eating, and neglect in reading and writing.

The rating scale as a whole had been shown to correlate 0.77 with errors on a letter cancellation test and 0.85 with a visual search task (in a group of 24 neglect subjects), while the presence or absence of neglect on the mobility subscale used in this study showed highly significant associations with the presence or absence of neglect on line crossing, letter cancellation, and visual search.

The patient's husband made these mobility subscale ratings daily for the duration of the study and over a 34 day follow up period. As the subject was greatly impaired on backward digit span this measure was included as a control for spontaneous remission of general cognitive capacities.

\section{Post-baseline measures}

The star cancellation and digit backward measures were repeated over five days, Monday to Friday of the following week. In addition, the subject's husband continued to rate mobility problems seven days a week during his two hour visit each afternoon.

The patient was discharged home shortly after the end of training. Star cancellation was followed up at three and six weeks after the end of training. The subject's husband kept daily ratings for 34 days after the end of the training period. These ratings could not be compared with the ratings in hospital because he was observing her for longer periods and hence was more likely, for instance, to report that she "often" collided with doorways at home than at hospital. His follow up mobility ratings are presented separately from those carried out in hospital.

\section{Training procedure}

As in case 2, the aim of training was to teach the subject to use the partially hemiparetic left arm as a perceptual anchor, thereby also increasing activation in the damaged hemisphere. The additional aspect of the training was the use of the "neglect alert device." This consists of a small metal box, approximately 12 $\times 8 \times 2 \mathrm{~cm}$, with a switch attached by a cable. The device can be set to emit a loud buzzing noise if the switch is not pressed within a predetermined, and variable, time interval. A red light mounted on the box remains on as long as the buzzer is not activated. Both box and switch are sufficiently light and portable and can be used in a variety of situations. The pressure required to activate the switch can also be adjusted on a continuous scale.

Training took place for a total of 10 hours, two hours a day for five days. A psychologist (NN) carried out the training for one hour each day and an occupational therapist carried out the training for the other hour. The subject was taught to place her arm to the left of a variety of activities. The neglect alert device and switch were placed to the left of the subject's left arm, and she was required to press the switch sufficiently frequently to prevent the buzzer going off. The time span allowed before the buzzer went off varied randomly between four and 18 seconds. During the training phase, testing always took place before the two afternoon training sessions, thus ensuring that any changes in behaviour were not attributable to the immediately preceding training session.

\section{Results}

The star cancellation errors reduced from a mean (SD) of $23.6(6.8)$ at baseline to a mean of $13.4(5.2)$ during training (fig $3, p<$ 0.02).

Errors at three and six weeks after training, were much lower than during the training (4 and 3 respectively, fig 3 ).

Ratings of mobility problems reduced from a mean of $16 \cdot 0(2 \cdot 2)$ at baseline to a mean of $11 \cdot 2(2 \cdot 2)$ during training (fig $4, p<0.02$ ). No significant difference between digits backward at baseline and after training was observed (2.8 $(0.45) v 3.0(0.71)$, fig 4$)$; suggesting that spontaneous remission was unlikely to have caused the observed changes in performance.

The husband's daily ratings of the patient's mobility after the end of training show deterioration in her neglect, which worsened until she attended the breast clinic where her cancer had been treated. At the appointment (about

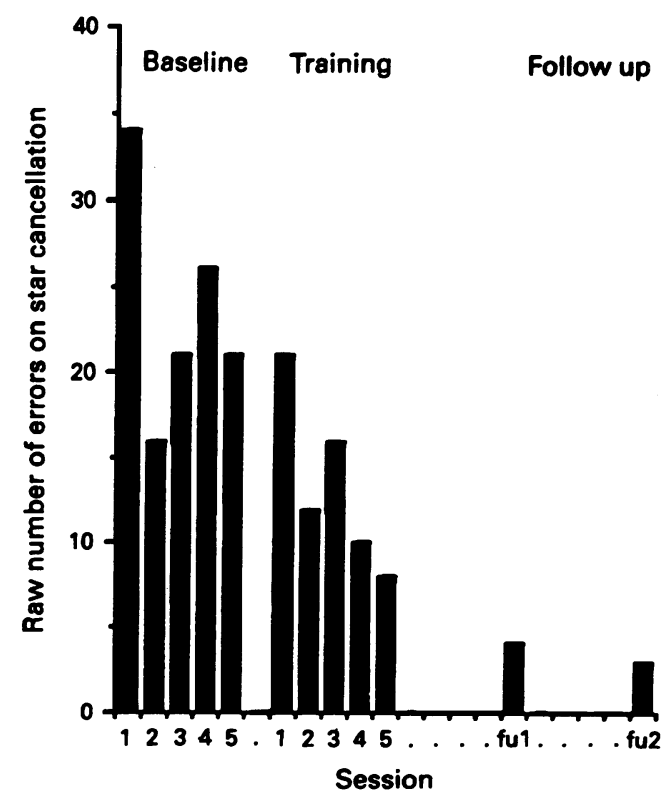

Figure 3 Case 2: star cancellation performance, at baseline, after training and at follow up. 


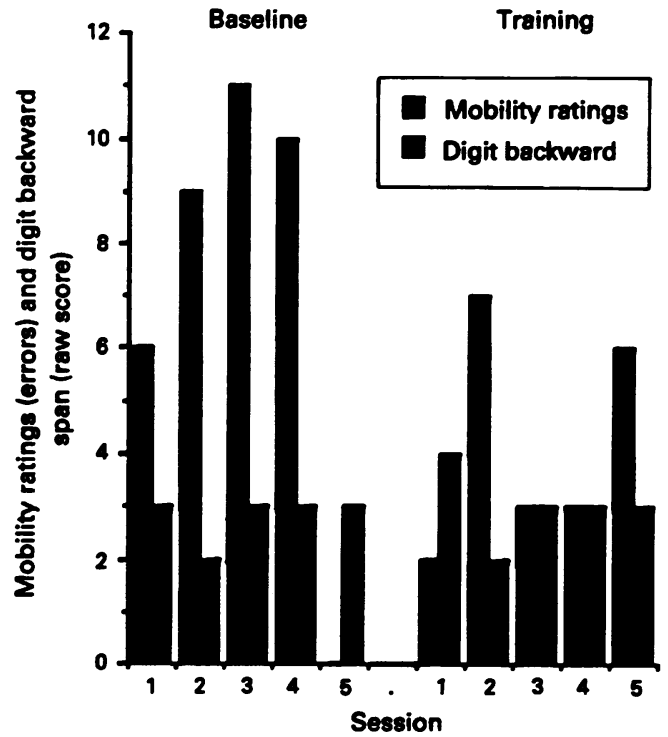

Figure 4 Case 2: mobility ratings and digit backward span, at baseline and after training.

which she had been increasingly worried) she was reassured that her cancer had, in her terms, been "cured" completely, and her neglect ratings steadily declined.

CASE 3

The treatment effects obtained in case 1 were replicated in case 2 , albeit that the arm activation was augmented by the neglect alert device. However, it was still not possible to disentangle arm activation factors from perceptual cueing factors. In case 3 we attempted to reduce the perceptual cueing aspect of the treatment in order to determine whether this eliminated the apparent efficacy of the treatment.

\section{Subject}

A previously fit 62 year old right-handed motor mechanic suffered a left hemiparesis and a left homonymous hemianopia. Seventeen days later he was fully oriented for time, place, and

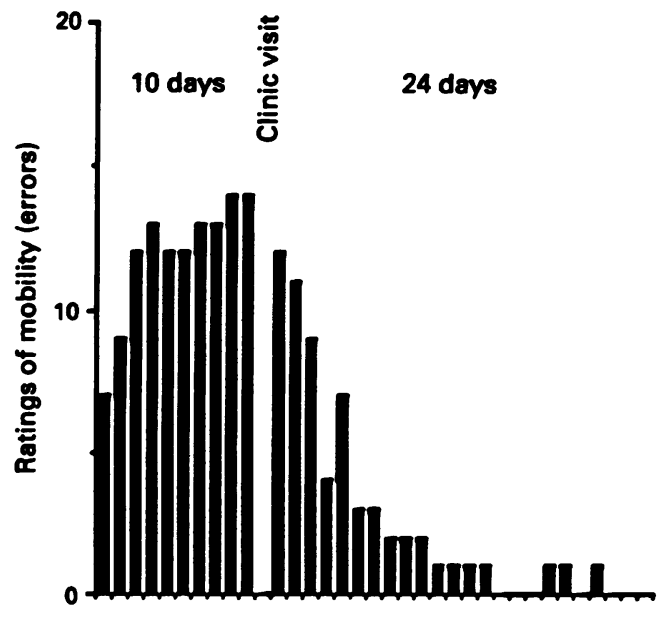

Figure 5 Case 2: mobility ratings during follow up period. person but showed clear left visual neglect; he was aware of and distressed by it. $\mathrm{He}$ also showed sensory neglect on his left side. His mini-mental state score was $28 / 30$. He showed $0 / 5$ power in his left arm, 4/5 in his left leg, and normal power in his right limbs. He showed decreased sensation in his left arm and leg. A CT scan showed multiple cerebral infarcts in the region of the right middle cerebral artery.

Therapists in the hospital noted unremitting left visual neglect leading to considerable difficulties with everyday life activities. Neuropsychological assessment five weeks after the stroke revealed severe left visual neglect, as measured by the "conventional" items of the behavioural inattention test (BIT), where he scored in the severe neglect range (54/146). Immediate and delayed logical memory on the revised Wechsler memory scale were both in the normal range for his age (57th and 64th centiles respectively), and he showed no significant perseveration on the Nelson version of the Wisconsin card sorting test (where the cards were aligned vertically rather than horizontally to minimise the effects of neglect; total errors, 11/48; number perseverative, 4/11). He scored in the normal range on the similarities and comprehension subtests of the Wechsler adult intelligence scale (revised), with (38th and 75 th age scaled centiles respectively). His digit span was also normal (eight digits forward and six backward). He was greatly impaired on object assembly and block design, however (< 1 and 10th centile respectively). His estimated premorbid IQ was 110 on the national adult reading test and he was not significantly depressed nor anxious (scores of 4 and 0 , respectively, on the hospital anxiety and depression scale).

\section{Baseline assessment}

Baseline measures were collected for six days, beginning five weeks after his stroke, and included the star cancellation subtest of the BIT. Another target measure was an adaptation of the star cancellation test relying only on touch, which the patient carried out blindfolded. The target small stars on the test were raised by placing several small discs of paper on each so that they could be felt by the patient. He was instructed to find and touch all the raised stars on the page. This was used to determine whether any treatment effects generalised to non-visual aspects of inattention.

The control measure was an adaptation of Benton's line orientation test, changed so that the lines to be selected were always in the right visual field. This is a measure of visuospatial functioning. A total of six baseline measures, five training measures, and three measures of return to baseline were taken over a period of 23 days. Follow up measures were taken at one, two, and three weeks after the end of the return to baseline period.

\section{Training procedure}

The training in this case consisted solely of left arm activation using the neglect alert device. No explicit instructions for perceptual anchoring were given, unlike the previous two cases. 
During the first half of the first training session the procedure was identical to that in case 2, with omission of the perceptual anchoring instructions. In spite of the patient's rating of $0 / 5$ power in his left arm, he did succeed, with prompting, in pressing the switch on his left with a clubbed left hand. However, it was noticed after 30 minutes of training that the patient seemed to press the switch of the device automatically, without attending to it, thereby preventing the buzzer from going off. Because this seemed to reduce the effect of the device on his neglect the procedure was changed for the remaining sessions to make the patient press the switch as soon as the buzzer went off, rather than trying to prevent it going off. The procedure then no longer resembled avoidance conditioning.

Training took place for a total of 10 hours, two hours a day for five days. A psychologist (NN) carried out the training for one hour each day and an occupational therapist carried out the training for the other hour. The subject was taught to carry out a number of tasks while simultaneously pressing the switch to his left as soon as the buzzer went off, the time between buzzes varying, as in the previous study.

During the training phase testing always took place before the two afternoon training sessions, thus ensuring that any changes in behaviour were not attributable to the immediately preceding training session. Assessment for the first three sessions of the baseline phase was carried out by a psychologist blind as to treatment regimen.

\section{Results}

The difference between baseline and training for star cancellation and star touch was significant (fig 6: star cancellation; $p<0.004$; star touch; $\mathrm{p}<0.003$ ). Baseline versus combined return to baseline and follow up scores (that is,

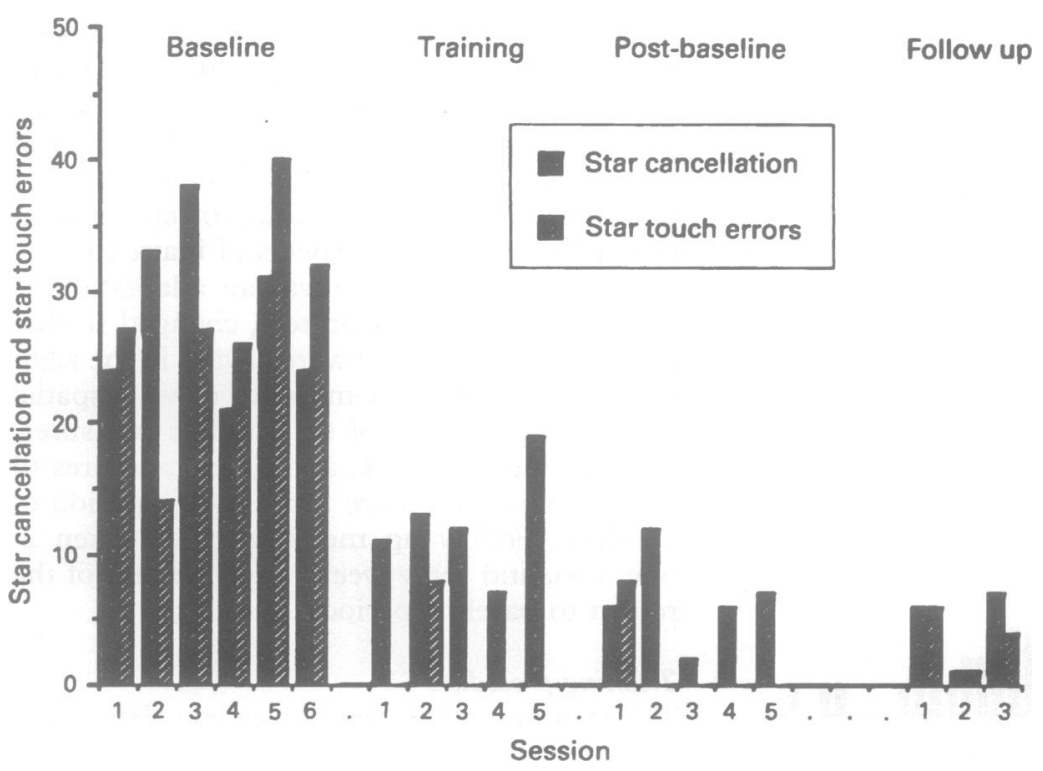

Figure 6 Case 3: star cancellation and star touch errors, at baseline, after training and at follow up.

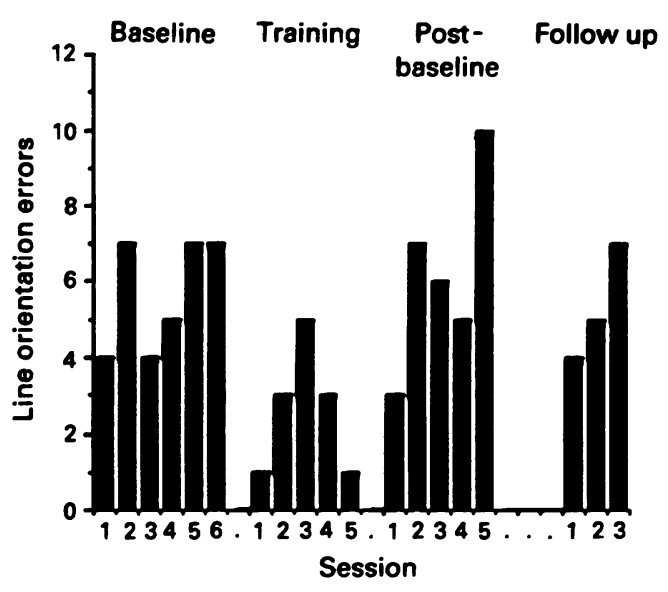

Figure 7 Case 3: line orientation errors, at baseline, after training and at follow up.

excluding the training period) was significant for both measures (star cancellation: $\mathrm{p}<$ 0.002 ; star touch: $\mathrm{p}<0.002$ ).

Errors were significantly reduced from baseline to training in the line orientation measure (fig $7, \mathrm{p}<0.02$ ), but a similarly significant increase in errors between training and the combined follow up period $(p<0.02)$ resulted in no significant difference between the baseline and follow up periods.

\section{Discussion}

Case 2's mobility ratings of neglect, as rated by her husband, appeared to fluctuate in accordance with her mood and anxiety. This may reflect attentional difficulties caused by these emotional variables compromising the execution of compensatory scanning strategies, 7 though other explanations are possible.

Case 3 seemed to show improvements in neglect similar to those shown in the previous two cases, even though the treatment consisted solely of the arm activation procedure without any explicit instructions for perceptual anchoring on the left arm. Thus the arm activation component of the previous two cases may have been the more effective component of treatment, though of course it is difficult to compare such different patients.

Regarding the control measures in case 3, the line orientation measure did, contrary to expectations, improve significantly during the training. That this is unlikely to reflect a spontaneous remission contaminating the results is shown by the fact that it returned to baseline levels after the end of treatment. It seems therefore that it was only a partial control measure and showed short term response to treatment rather than a long term response. Though only right sided lines were used in this test, neglect may have affected performance in right hemispace, and this neglect may have been sensitive to short term effects of treatment. The lack of significant change in perseveration confirms the conclusion that spontaneous remission could not have influenced the apparent treatment effects. 
The question posed in the introduction was whether left arm activation or perceptual anchoring, or both, can produce enduring and therapeutic improvements in neglect. The answer from the three case studies presented above is a qualified yes, at least for several weeks after treatment-any effects in terms of months or years are unknown.

Though this conclusion has important clinical implications, the theoretical issues are perhaps more interesting. Is there something special about cueing attention to the left by moving the arm on the side opposite the lesion, or is it simply that the arm is reliably present in the person's daily environment and therefore more consistently elicits left scanning responses taught during training?

The results of the three cases cannot be directly compared, but it is interesting to note that case 3 seemed to show the kinds of improvements shown by cases 1 and 2 in the absence of explicit instructions on perceptual anchoring. Of course the light and switch to the left cued leftward responses, but he was not taught explicitly to "find" his left arm in the way the first two patients were.

This conclusion is supported by a more recent and more rigorous study. Robertson and North experimentally tested which factors determined the improved performance of this patient. ${ }^{14}$ This study found that visual cues were irrelevant to the improved performance: even when the patient made left hand responses behind a screen, his neglect still decreased. The study also showed that the left finger movements had to be made in left hemispace: neither left hand movements in right hemispace nor right hand movements in left hemispace produced a reduction in neglect. This suggests that the potent effect of the treatment in the present three case studies was activation of the left arm in left hemispace and that the visual anchoring or scanning aspect of the treatment was irrelevant. If this is the case then these results have profound implications for how unilateral neglect is managed and treated in rehabilitation units.

Though the literature on rehabilitation of neglect has suggested positive therapeutic effects of scanning based on visual treatment, ${ }^{15-17}$ a review of this literature shows that treatment gains tended to occur on test materials strongly similar to the training materials. ${ }^{7}$ This conclusion is supported, for instance, by an attempt to replicate, using single case designs, some of the New York rehabilitation studies. ${ }^{18}$ This study found that scanning training using a "light board" (detecting lights on a $2 \mathrm{~m}$ wide board) produced improvements on this measure but not on cancellation tests, while training in cancellation-type procedures resulted in improvements in cancellation tests but not on light board performance. Furthermore, Robertson et al found by using computerised training programmes that where the training procedures share few, if any, stimulus characteristics with the test procedures no effects of treatment were observed. $^{19}$

One explanation of the possible beneficial effects of activating motor responses in the hemiplegic side in unilateral neglect stems from the evidence that there exist several independent spatial frames of reference, some of which may be selectively spared in cases of unilateral neglect. ${ }^{5}$ A recent study has shown this in a case where the subject showed significant unilateral neglect in "reaching space", but not in "locomotor space" when bisecting lines with a laser beam, the targets being far outside normal reaching space. ${ }^{20}$

The effect of motor responses on the neglected side may therefore make the representational schema for the left side of the body more salient, which in turn activates or increases attention to the left side of reaching space. This suggestion must await further detailed experimental investigation.

Technical specifications of the neglect alert device are available from C Geggie, Bioengineering Unit, Princess Margaret Rose Hospital, Fairmilehead, Edinburgh, Scotland

We thank the patients who participated in this research, as well as our colleagues at Astley Ainslie Hospital who helped us execute the research. In particular, we would like to thank Sue MacKenzie, Jane Hornby, Pauline Ritchie, Maureen McKail, and Brian Pentland for their help and support.

1 Joanette $Y$, Brouchon $M$. Visual allesthesia in manual pointing: some evidence for a sensori-motor cerebral organization. Brain and Cognition 1984;3:152-65.

2 Obersteiner H. On allochiria. Brain 1882;4:153-63.

3 Joanette Y, Brouchon M, Gauthier L, Samson M. Pointing with left versus right hand in left visual field neglect. Neuropsychologia 1986;24:391-6.

4 Halligan PW, Marshall JC. Laterality of motor response in visuo-spatial neglect: a case study. Neuropsychologia 1989; 27:1301-7.

5 Rizzolatti G, Camarda R. Neural circuits for spatial attention and unilateral neglect. In: Jeannerod M, ed. Neurophysiological and neuropsychological aspects of neglect. physiological and neuropsychological

6 Halligan PW, Manning L, Marshall JC. Hemispheric activation vs spatio-motor cueing in visual neglect: a case study. Neuropsychologia 1991;29:165-76.

7 Robertson I. The rehabilitation of attentional and hemiinattentional disorders. In: Humphreys $G$, Riddoch J, eds. Cognitive neuropsychology and cognitive rehabilitation. London: Lawrence Erlbaum, (in press).

8 Robertson I. Digit span and visual neglect: a puzzling relationship. Neuropsychologia 1990;28:217-22.

9 Robertson I. Use of left versus right hand in responding to lateralised stimuli in unilateral neglect. Neuropsychologia 1991;29:1 129-35.

10 Diller L, Ben-Yishay Y, Gerstman L, Goodkin R, Gordon $\mathrm{W}$, Weinberg J. Studies in cognition and rehabilitation in hemiplegia. New York: New York University Medical hemiplegia. New York: New York University Medic

11 Halligan PW, Cockburn J, Wilson BA. The behavioural assessment of visual neglect. Neuropsychological Rehabilitation 1991;1:5-32.

12 Wilson B, Cockburn J, Halligan P. Behavioural inattention test. Fareham. Thames Valley Test Company, 1988.

13 Keenan E. A study of the relationship between performance on perceptual tasks and corresponding difficulties in the everyday lives of patients with right hemisphere damage [dissertation]. Leicester: British Psychological Society, 1986.

14 Robertson I, North N. Spatio-motor cueing in unilateral left neglect: the role of hemispace, hand and activity in 1992;30:553-63.

15 Weinberg, Diller L, Gordon W, Gerstman L, Lieberman A Lakin $\mathrm{P}$, et al. Visual scanning training effect on readingLakin $P$, et al. Visual scanning training effect on reading-
related tasks in acquired right brain damage. Arch Phys related tasks in acquired rig

16 Weinberg M, Diller L, Gordon W, Gerstman L, Lieberman A, Lakin P, et al. Training sensory awareness and spatial organisation in people with right brain damage. Arch Phys Med Rehabil 1979;60:491-6.

17 Young G, Collins D, Hren M. Effect of pairing scanning training with block design training in the remediation of perceptual problems in left hemiplegics. Fournal of Clinical Neuropsychology 1983;5:201-12.

18 Gouvier W, Bua B, Blanton P, Urey J. Behavioural changes following visual scanning training: observation of five cases. International fournal of Clinical Neuropsychology 1987;9:74-80.

19 Robertson I, Gray J, Pentland B, Waite L. A randomised controlled trial of computer-based cognitive rehabilitation for unilateral left visual neglect. Arch Phys Med Rehabil 1990;71:663-8.

20 Halligan PW, Marshall JC. Left neglect for near but nor far space in man. Nature 1991;350:498-500. 